\title{
Neonatal Meningitis with Septicemia by Elizabethkingia meningoseptica: A Case Report
}

\author{
Neetha S. Murthy ${ }^{1} \quad$ Sowmya G. Shivappa $^{1} \quad$ A. Tejashree $^{1} \quad$ Krishna M.V.S. Karthik $^{1} \quad$ R. Deepashree ${ }^{1}$ \\ ${ }^{1}$ Department of Microbiology, JSS Medical College, Mysuru, \\ Karnataka, India \\ J Lab Physicians 2022;14:362-364.

\begin{abstract}
Address for correspondence Sowmya G. Shivappa, MBBS, MD, Department of Microbiology, JSS Medical College, JSS AHER, Sri Shivarathreeshwaranagar, Mysuru, 570015, Karnataka, India
\end{abstract} \\ (e-mail: sowmyashivappa@gmail.com).
}

\begin{abstract}
Keywords

- septicemia

- Elizabethkingia meningoseptica

- meningitis

- multidrug resistant

- neonate
\end{abstract}

\section{Introduction}

Elizabethkingia meningoseptica is an emerging cause of lifethreatening nosocomial infections among neonates. ${ }^{1}$ This opportunistic pathogen was primarily placed under genus Flavobacterium (1959) and later moved to genus Chryseobacterium (1994). Eventually in the year 2005 based on 16s rRNA phylogenetic studies, this aerobic bacillus was classified in the genus Elizabethkingia (an eponym from its discoverer Elizabeth O. King). ${ }^{2}$ E. meningoseptica is a gramnegative bacillus that is nonmotile, nonfermentative bacteria capable of splitting tryptophan to produce indole and is oxidase positive. Whole genome sequence analysis has established the intrinsic ability of this microbe to form biofilms and serve as a potential stockpile of novel- $\beta$-lactamase genes. ${ }^{3,4}$

published online February 9, 2022
DOI https://doi.org/ $10.1055 / \mathrm{s}-0042-1742635$ ISSN 0974-2727.
Literature documents sparse case reports of E. meningoseptica from India and around the world. Few cases of wound infections, keratitis, nosocomial septicemia, nosocomial pneumonia, endocarditis, and meningitis in immunocompromised adults and preterm neonates have been reported globally., Several culpable sources of this opportunistic pathogen such as contaminated water supply, equipment tubing, infant formulas, and saline solutions have been documented apart from the environmental niches. ${ }^{7}$ Yet another remonstrance with respect to this bacillus is its habitual multidrug resistance with no established Clinical and Laboratory Standards Institute drug breakpoints. ${ }^{8}$ Given the above circumstances, empirical therapy and treatment standardization with respect to $E$. meningoseptica is a farfetched dream. We report a case of neonatal meningitis with septicemia caused by E. meningoseptica in an outborn term neonate. (c) 2022. The Indian Association of Laboratory Physicians. All rights reserved.

This is an open access article published by Thieme under the terms of the Creative Commons Attribution-NonDerivative-NonCommercial-License, permitting copying and reproduction so long as the original work is given appropriate credit. Contents may not be used for commercial purposes, or adapted, remixed, transformed or built upon. (https://creativecommons.org/ licenses/by-nc-nd/4.0/)

Thieme Medical and Scientific Publishers Pvt. Ltd., A-12, 2nd Floor, Sector 2, Noida-201301 UP, India 


\section{Case Report}

We present a case of 1-day-old term outborn infant with $E$. meningoseptica attributed meningitis and septicemia presenting with convulsions. A single live, full-term, male baby delivered by normal vaginal delivery at a peripheral district hospital presented with birth asphyxia. The APGAR (appearance, pulse, grimace, activity, and respiration) score at birth unknown, the baby presented to our tertiary care center with two to three episodes of convulsions, sepsis screen positive, and respiratory distress. The neonate was stabilized with a loading dose of phenobarbitone $20 \mathrm{mg} / \mathrm{kg}$ followed by a maintenance dose of $10 \mathrm{mg} / \mathrm{kg}$. Injection calcium was given $2 \mathrm{~mL} / \mathrm{kg}$ and the child was shifted to the neonatal intensive care for further management.

At admission, the child was moderately active with a heart rate of 146/minute and respiratory rate of $93 /$ minute. Weight of the neonate was $2.88 \mathrm{~kg}$. The ultrasound brain yielded a normal study with no intracranial bleed, brain parenchymal lesions, or ventricular enlargement. The cerebral hemispheres, basal ganglia, corpus callosum, and posterior fossa structures appeared normal. Magnetic resonance imaging brain showed mild hypoxic ischemic encephalopathy.

The blood investigations showed a raised total cell count $(128,900)$ and reduced platelet count $(67,000)$. C-reactive protein was raised with a value of 27.91. Creatinine was found to be $1.32 \mathrm{mg} / \mathrm{dL}$. Liver function parameters were within normal limits. Arterial blood gas analysis at admission showed metabolic acidosis with compensatory respiratory alkalosis. Cerebrospinal fluid (CSF) analysis at admission showed predominant neutrophils with normal protein and sugar levels.

Blood sample ( $4 \mathrm{~mL}$ ) from the neonate ${ }^{8}$ was inoculated into a culture bottle and incubated in BacT/ALERT Microbial Colorimetric Detection System. Sample flagged positive in 23 hours. Flagged sample was subjected to direct gram staining and subsequently plated on MacConkey along with $5 \%$ sheep blood agar. Gram smear showed gram-negative bacilli and culture

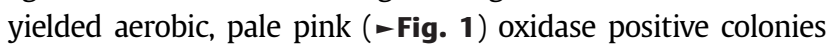
on MacConkey agar and nonhemolytic colonies on blood agar. Culture isolate smear showed gram-negative bacillus in concordance with the direct smear findings (- Fig. 2). VITEK-2 (bioMérieux) identification yielded E. meningoseptica with $99 \%$ probable confidence of identification. CSF sample from the child was subjected to automated culture (BacT/ALERT System). CSF culture also yielded $E$. meningoseptica by the VITEK 2 system with a $99 \%$ identification confidence. Both the blood and CSF isolate showed resistance to $\beta$-lactams, carbapenems, and aminoglycosides. Second- and third-generation quinolones were effective with an in vitro minimal inhibitory concentration of $0.5 \mu \mathrm{g} / \mathrm{mL}$. The child was started on parenteral ciprofloxacin along with symptomatic management. In vivo response to therapy was clinically admirable and the child was discharged on day 7 post treatment initiation with absolutely no residual morbidity.

\section{Discussion}

Genus Elizabethkingia houses a notorious bunch of rare opportunistic pathogens responsible for multidrug resistant

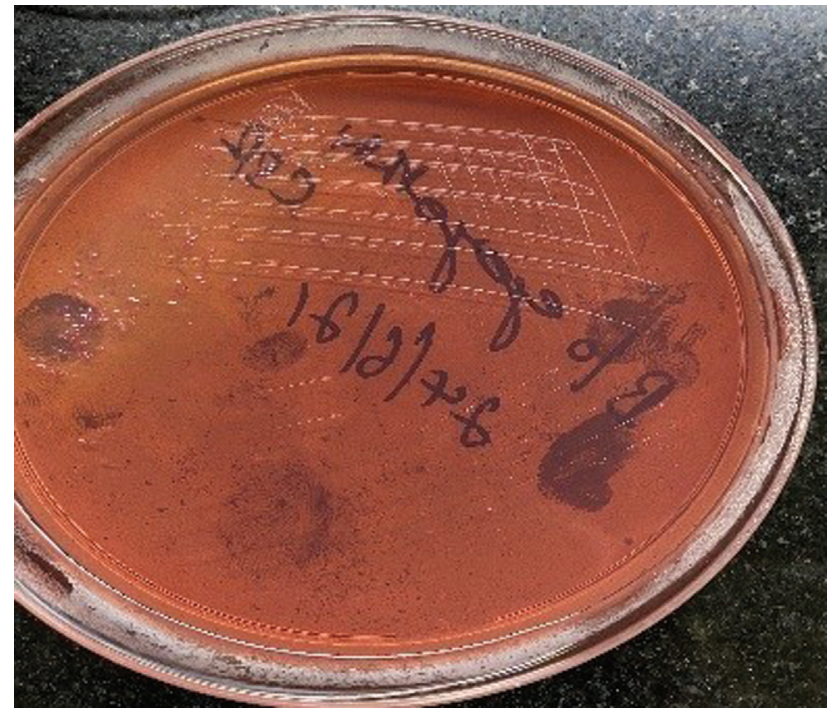

Fig. 1 Pale pink colonies on MacConkey agar.

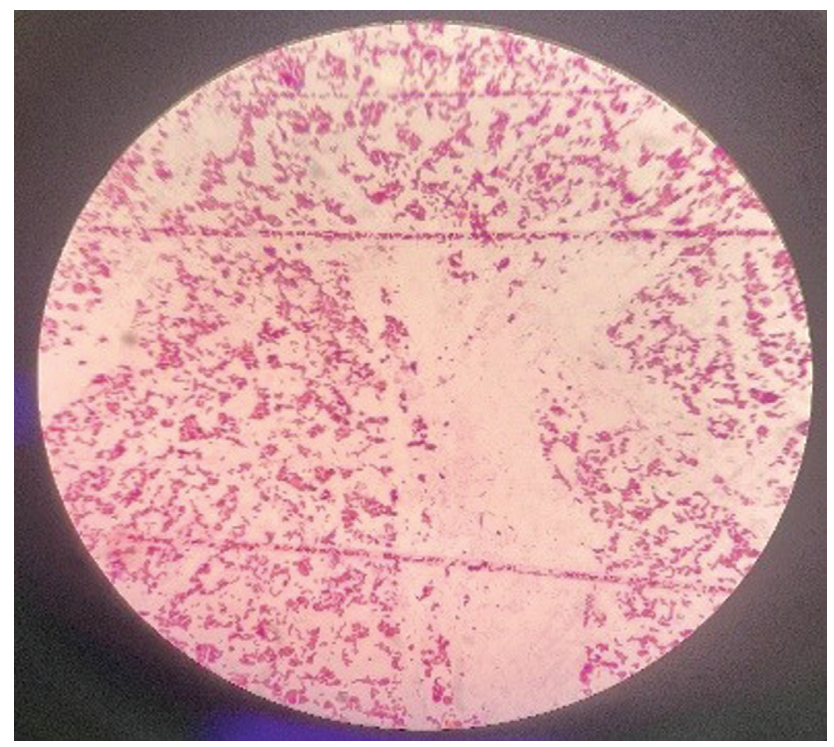

Fig. 2 Smear from culture showing gram-negative bacilli.

lethal infections. The mortality rate associated with this rare pathogen is around $23 \%{ }^{5,9}$ Essentially the genus is composed of saprophytic bacteria capable of survival in chlorinated water, hospital equipment, and pediatric nurseries. ${ }^{10}$ Interestingly although deemed to be an opportunistic pathogen commonly infecting the immunocompromised subjects, Elizabethkingia species are not part of the normal human microbial flora. The bacteria belonging to this genus are known to possess virulence attributes such as proteases, catalases, acetyltransferases, peroxidases, heat shock proteins, capsular polysaccharide, and lipooligosaccharides. ${ }^{11}$ The adherence ability of E. meningoseptica NCTC $10016^{\mathrm{T}}$ onto abiotic surfaces such as intravascular devices via formation of biofilms has been well established. ${ }^{12}$ Multiple possible pathways of exposure and mechanisms of 
pathogenesis have been contemplated. However, exact mechanism of pathogenesis remains obscure. ${ }^{11}$

Considering the fact that bacterial species belonging to the genus Elizabethkingia are usually multidrug resistant, laboratory affirmed identification is of prime importance. On the laboratory front, strain-dependent variabilities ${ }^{2}$ in culture growth make manual identification unreliable. Commercial automated microbial identifications systems provide accurate and quick genus level identification of Elizabethkingia species. However, Elizabethkingia reference databases on commercial microbial identification systems find partial concordance with 16S rRNA gene sequencing-based specific species identification. ${ }^{13,14}$ Advances in automation and microbiological diagnostics bring with it the advantage of early diagnosis and better detection rates of Elizabethkingia species, thereby averting inappropriate antimicrobial therapy and outbreak prevention.

Members of the genus Elizabethkingia are by and large resistant to antimicrobials such as tetracycline, chloramphenicol, extended-spectrum $\beta$-lactams, and aminoglycosides. Biologically plausible chromosome and plasmid mediated $\beta$-lactam resistance due to Ambler class $D$ extended-spectrum serine- $\beta$-lactamase coding $b l a_{\mathrm{CME}}$ genes and carbapenem resistance due to $b l a_{\mathrm{B}}$ (subclass $\mathrm{B} 1$ ) and $b l a_{\mathrm{GOB}}$ (subclass B3) genes have been documented. ${ }^{15}$ Fluoroquinolones exhibit uniform volume distribution and better penetration of the blood brain barrier by virtue of their lipophilic nature. ${ }^{15,16}$ The child in our case report also demonstrated clinical improvement with 7 days of parenteral ciprofloxacin therapy and was discharged on day 8 of admission with no documented untoward incidents.

\section{Conclusion}

E. meningoseptica is a difficult to diagnose saprophytic nonfermenter with a handful of cases being reported from pediatric nurseries and critical care units in the recent times. This aggrandize with respect to notification of E. meningoseptica infections is attributable to the availability of rapid, accurate, commercial identification systems for laboratory diagnosis. In conclusion, given the independent attributable mortality/morbidity and multidrug resistance profile of Elizabethkingia, development of robust standard infection control practices to combat this emerging pathogen is inevitable. Armed with automated identification systems, the microbiology laboratory can combat the formidable rapid identification challenge paving way toward early appropriate treatment initiation, resulting in favorable patient outcome as documented in our case report.

\section{Funding}

No separate funding was received apart from using laboratory facilities as part of the routine laboratory support from JSS medical college.
Conflict of Interest

None declared.

\section{References}

1 Long SS. Principles and Practice of Pediatric Infectious Diseases. Fourth edition; 2012:1547-1712

2 Kim KK, Kim MK, Lim JH, Park HY, Lee S-T. Transfer of Chryseobacterium meningosepticum and Chryseobacterium miricola to Elizabethkingia gen. nov. as Elizabethkingia meningoseptica comb. nov. and Elizabethkingia miricola comb. nov. Int J Syst Evol Microbiol 2005;55(Pt 3):1287-1293

3 Matyi SA, Hoyt PR, Hosoyama A, Yamazoe A, Fujita N, Gustafson JE. Draft genome sequences of Elizabethkingia meningoseptica. Genome Announc 2013;1(04):e00444-e13

4 Chen S, Soehnlen M, Downes FP, Walker ED. Insights from the draft genome into the pathogenicity of a clinical isolate of Elizabethkingia meningoseptica Em3. Stand Genomic Sci 2017; $12: 56$

5 Bhat KS, Priya R, Krishnan L, Kanungo R. Elizabethkingia meningoseptica bacteremia in a neonate: a case report and mini-review of the literature. J Curr Res Sci Med 2016;2:42-45

6 Amer MZ, Bandey M, Bukhari A, Nemenqani D. Neonatal meningitis caused by Elizabethkingia meningoseptica in Saudi Arabia. J Infect Dev Ctries 2011;5(10):745-747

7 Rastogi N, Mathur P, Bindra A, et al. Infections due to Elizabethkingia meningoseptica in critically injured trauma patients: a seven-year study. J Hosp Infect 2016;92(01):30-32

8 Kellogg JA, Manzella JP, Bankert DA. Frequency of low-level bacteremia in children from birth to fifteen years of age. J Clin Microbiol 2000;38(06):2181-2185

9 Lin YT, Chiu CH, Chan YJ, et al. Clinical and microbiological analysis of Elizabethkingia meningoseptica bacteremia in adult patients in Taiwan. Scand J Infect Dis 2009;41(09): 628-634

10 Steinberg JP, Burd EM. Other gram negative and gram variable bacilli. In: Mandell GL, Bennett JE, and. Dolin R, eds. Mandell, Douglas and Bennett's Principles and Practice of Infectious Diseases. Seventh edition. United States: Churchill Livingstone; 2010:3015-3033

11 Chen S, Soehnlen M, Blom J, Terrapon N, Henrissat B, et al. Comparative genomic analyses reveal diverse virulence factors and antimicrobial resistance mechanisms in clinical Elizabethkingia meningoseptica strains. PLOS ONE 2019;14(10):e0222648

12 Jacobs A, Chenia HY. Biofilm formation and adherence characteristics of an Elizabethkingia meningoseptica isolate from Oreochromis mossambicus. Ann Clin Microbiol Antimicrob 2011; 10:16

13 Lau SKP, Chow W-N, Foo C-H, et al. Elizabethkingia anophelis bacteremia is associated with clinically significant infections and high mortality. Sci Rep 2016;6:26045

14 Lin J-N, Lai C-H, Yang C-H, Huang Y-H, Lin H-F, Lin H-H. Comparison of four automated microbiology systems with 16S rRNA gene sequencing for identification of Chryseobacterium and Elizabethkingia species. Sci Rep 2017;7(01):13824

15 Huang YC, Lin YT, Wang FD. Comparison of the therapeutic efficacy of fluoroquinolone and non-fluoroquinolone treatment in patients with Elizabethkingia meningoseptica bacteraemia. Int J Antimicrob Agents 2018;51(01):47-51

16 Vincent JL, Bassetti M, François B, et al. Advances in antibiotic therapy in the critically ill. Crit Care 2016;20(01):133 